\title{
O COMPORTAMENTO SEXUAL DOS DEPENDENTES DE COCAÍNA/CRACK E A VULNERABILIDADE EM RELAÇÃO ÀS DSTS EM UMA COMUNIDADE TERAPÊUTICA
}

\author{
THE SEXUAL BEHAVIOR OF COCAINE / CRACK DEPENDENTS \\ AND VULNERABILITY IN RELATION TO STDS IN A THERAPEUTIC \\ COMMUNITY
}

\author{
Fabiane de Jesus Lacerda ${ }^{1}$, Andréa Hofmeister da Nóbrega ${ }^{1}$, Beatriz Essenfelder \\ Borges $^{1,2^{*}}$, Daniel de Christo ${ }^{1}$ \\ ${ }^{1}$ Faculdades Integradas Santa Cruz de Curitiba, Curitiba, Paraná, Brasil \\ ${ }^{2}$ Faculdades Pequeno Príncipe de Curitiba, Curitiba, Paraná, Brasil \\ *Autor correspondente: Beatriz Essenfelder Borges. Rua Amazonas 677, Água Verde, \\ Curitiba. Telefone: 41 999975311. Email: biaessenfelder@gmail.com
}

\begin{abstract}
RESUMO
Esta pesquisa foi desenvolvida com o objetivo de identificar e descrever o comportamento sexual dos usuários de cocaína e/ou crack e a sua vulnerabilidade em relação às doenças sexualmente transmissíveis (DSTs), realizada nas dependências de uma Comunidade Terapêutica (CT), da Região Metropolitana de Curitiba. Sendo um estudo transversal, com amostra de 30 dependentes de cocaína e/ou crack, em tratamento, sob regime de abstinência, cuja coleta de dados feita por meio de entrevista individual e aplicação de questionário estruturado. Os dependentes, em tratamento na $\mathrm{CT}$, de maioria do sexo masculino, adultos e com média escolaridade. Os achados apontam que 33,3\% da amostra apresenta histórico de doença sexualmente transmissível, sendo 26,7\% desenvolvidas após o início do uso de drogas. As DSTs encontradas se apresentam em proporções de $10 \%$ para gonorreia; $6,6 \%$ para sífilis; 3,3\% para HIV e $13,3 \%$ para outras doenças sexualmente transmissíveis. Os resultados destacam a vulnerabilidade dos usuários em relação à contaminação por doenças sexualmente transmissíveis, diante da baixa adesão ao uso de preservativos e diversos outros comportamentos de risco, associados ao uso de substâncias psicoativas.
\end{abstract}

Palavras-chave: Usuários de drogas; cocaína; crack; vulnerabilidade em saúde; doenças sexualmente transmissíveis.

\begin{abstract}
Research developed to identify and describe the sexual behavior of cocaine and / or crack users and their vulnerability to sexually transmitted diseases (STDs). Developed in the dependencies of a Therapeutic Community (CT) of the Metropolitan Region of Curitiba. A cross-sectional study with a sample of 30 cocaine and / or crack dependents undergoing abstinence treatment. Data collection through individual interview and application of a structured questionnaire. The dependents undergoing treatment at TC are mostly males, adults and with average schooling. The findings indicate that $33.3 \%$ of the sample has a history of sexually transmitted disease, $26.7 \%$ of which were developed after the start of drug use. The STDs were present in proportions of $10 \%$ for gonorrhea, $6.6 \%$ for syphilis, $3.3 \%$ for HIV and $13.3 \%$ for other sexual diseases. The results highlight the vulnerability of users regarding the contamination of sexually transmitted diseases, due to the low adherence to the use of condoms and several other risk behaviors associated to the use of psychoactive substances.
\end{abstract}

Keywords: Drug users; crack; cocaine; vulnerability in health; sexually transmitted diseases. 


\section{INTRODUÇÃO}

Ao analisar a história da humanidade é visível que o consumo de substâncias psicoativas sempre esteve presente e nem sempre foi um problema social. Os seres humanos, constantemente, buscam alterações no nível de consciência, alívio de dores e prazer, por meio do uso de tais substâncias ${ }^{1}$.

Atualmente, o conceito mais utilizado para a definição do termo, droga, é o praticado pela Organização Mundial de Saúde (1993, 69-82), em que é definida como "qualquer substância natural ou sintética que, quando administrada por qualquer via, no organismo, pode modificar sua estrutura ou função"2.

O consumo dessas substâncias estabeleceu-se entre a maioria dos grupos sociais ao longo do tempo, passando por evoluções em suas composições e escalas de produção. As drogas passaram a ser consideradas um problema de saúde pública, a partir de 1980, devido ao aumento do consumo, danos à saúde dos usuários e problemas sociais, relacionados ao uso e abuso ${ }^{3}$.

A dependência de drogas é caracterizada pela associação de eventos comportamentais, cognitivos e fisiológicos que se manifestam após o frequente uso de um elemento psicoativo, acompanhado da necessidade incontrolável de consumo, aumento da tolerância, abstinência física e psicológica² .

$\mathrm{O}$ aumento do consumo de drogas tem impacto direto no meio econômico e social, tornando-se um desafio para os profissionais de saúde, familiares e para as políticas públicas de prevenção ao uso de drogas. O consumo cada vez mais precoce resulta no aumento dos quadros de abuso e dependência química ${ }^{4}$

Um estudo realizado em 2012 revelou que, em média, 230 milhões de pessoas, no mundo, fizeram uso de drogas ilícitas, no ano de 2010, sendo esse fenômeno responsável por vinte dos maiores fatores de risco para os problemas de saúde pública ${ }^{5}$.

No Brasil, os estudos revelam que $23 \%$ da população, entrevistada, já utilizou drogas ilícitas durante a vida e que as drogas estimulantes estão entre as mais consumidas. Em meio às drogas estimulantes ilícitas, a cocaína e derivados dela, como o crack, destacam-se devido aos problemas sociais e psicológicos, ocasionados pelo uso em qualquer uma de suas formas de consumo ${ }^{6}$.

A Erythroxylum coca, nome científico dado à planta da qual a cocaína é extraída, vem sendo consumida por décadas, pelos andinos. Os estímulos de funcionamento acelerado do sistema nervoso central (SNC), ocasionados pelo hábito de mascar as folhas, foram transmitidos entre gerações, sofrendo modificações e chegando às condições atuais e variadas formas de consumo ${ }^{7}$.

A cocaína chega ao consumidor de três maneiras: na forma de sal, cloridrato de cocaína ou em forma de pó, podendo ser aspirado ou dissolvido para uso endovenoso, ou ainda, transformado em crack. A produção do crack ocorre a partir do cloridrato de cocaína, dissolvido em água e da adição de um agente alcalino (hidróxido de sódio ou bicarbonato de sódio), que aquecido forma a "pedra" fumada em cachimbos ${ }^{8}$.

O uso da cocaína e do crack, assim como de todas as outras substâncias psicoativas, é cercado pela criminalidade e pela violência, oriundos do mercado das drogas, ocasionando danos devastadores à economia e ao meio social. Os usuários, em resposta às suas práticas e exposição aos riscos, estão suscetíveis a diversas complicações, dentre elas, à contaminação por doenças sexualmente transmissíveis ${ }^{9}$.

Pesquisas relacionadas ao comportamento humano e à ação das drogas, no organismo, demonstram que a exposição à violência e às condições precárias de sobrevivência, normalmente associadas ao uso de drogas, são fatores que contribuem diretamente nas ações e nas medidas preventivas, adotadas pelo indivíduo durante suas relações sexuais ${ }^{10}$.

A regulamentação de procedimentos e condutas, para os usuários de drogas que procuram atendimento nos serviços de saúde, possibilita identificar a contaminação por HIV ou outras DSTs. Esse procedimento viabiliza uma estimativa acerca do número de indivíduos, contaminados por algum tipo de doença sexualmente transmissível, possibilitando diminuir a carência de dados relacionados ao padrão de consumo de drogas, aos meios utilizados para obtê-las e sobre as doenças sexualmente transmissíveis ${ }^{11}$.

A implementação de medidas relacionadas, ao combate ao uso de drogas e captação de informações relativas ao consumo e comportamento adotados pelos usuários, são essenciais para a criação de ferramentas efetivas no tratamento da dependência química e reinserção do indivíduo na sociedade, uma vez que o fracasso, neste processo, gera um alto índice de retomada ao uso de drogas e o aumento da transmissão e agravo das doenças sexualmente transmissíveis ${ }^{12}$. 
Reconhecendo as dificuldades no combate ao uso e no tratamento dos usuários, a Política Nacional sobre drogas do Governo Federal estabeleceu parcerias com instituições da sociedade, que oferecem serviços à comunidade, na área de dependência química, disponibilizando tratamento em regime de residência e buscando a reinserção do indivíduo à sociedade ${ }^{11}$.

A comunidade terapêutica é um modelo de tratamento residencial, oferecido aos dependentes químicos, em que se utiliza do método da experiência de vida e de convivência, em uma cultura organizada, abordando aspectos sociais, culturais e religiosos para compreender a magnitude dos problemas dos usuários e identificar a melhor forma de intervenção, diminuindo o número de reincidência ${ }^{13}$.

Diante das condições atuais de consumo de substâncias psicotrópicas e o aumento no número de casos de contaminação, por doenças sexualmente transmissíveis, levantamos o questionamento: Os dependentes de cocaína e/ou crack são, ou não, mais vulneráveis à contaminação por DSTs? Diante disso, o desenvolvimento da presente pesquisa justifica-se, em virtude da necessidade de investigar a possível vulnerabilidade dessa população usuária de cocaína/crack, a fim de servir como base à criação de medidas capazes de prestar assistência e diminuir o índice de contaminação, por doenças sexualmente transmissíveis, em meio aos usuários das substâncias supracitadas.

\section{METODOLOGIA}

Pesquisa transversal não controlada, descritiva e quantitativa. Foi realizada nas dependências de uma residência terapêutica, localizada na Região Metropolitana de Curitiba, após estar de acordo com a resolução 466, de 12 de dezembro de 2012, no que diz respeito aos critérios éticos. Aprovada pelo Comitê de Ética e Pesquisa do Instituto Paranaense de Otorrinolaringologia, sob parecer consolidado pelo número CAAE 76251317.8.0000.5529.

A amostra foi constituída por 30 indivíduos, os quais atenderam a todos os critérios de inclusão e exclusão aplicáveis. Foram incluídos, neste estudo, dependentes de cocaína/crack, de ambos os sexos, com idade igual ou superior a 18 anos, inseridos no processo de tratamento, sob regime de abstinência. Os indivíduos que não atenderam aos critérios supracitados foram excluídos da pesquisa.
Os participantes receberam, individualmente, o Termo de Consentimento Livre e Esclarecido (TCLE), sendo disponibilizado tempo adequado à leitura e compreensão do termo. Aos participantes que apresentaram dificuldades, foram realizados os devidos esclarecimentos pela pesquisadora acadêmica. Após a assinatura do TCLE, concordando com a metodologia proposta pelos pesquisadores.

Os indivíduos receberam um questionário estruturado, previamente elaborado, composto por questões relacionadas ao comportamento, com variáveis como: sexo do indivíduo, faixa etária, nível de escolaridade, frequência de prática sexual, número de parceiros sexuais, uso de preservativo, prática sexual sob efeito de cocaína/crack, uso de preservativo nas relações sob efeito de entorpecentes, prostituição relacionada ao uso de substâncias e histórico de doenças sexualmente transmissíveis.

Os dados obtidos, através do questionário estruturado, foram analisados e submetidos a testes estatísticos de frequência, pelo programa estatístico IBM SPSS, versão 2.2. O nível de significância adotado foi de $\mathrm{p}<0,05$.

\section{RESULTADOS}

Em relação ao sexo dos participantes, no que diz respeito à dependência de cocaína/crack, observou-se maior incidência do sexo masculino, com proporções de $93,3 \%$ e baixa incidência do sexo feminino, com índice de 6,7\%. (Gráfico1).

GRÁFICO 1. Sexo dos dependentes de cocaína/crack

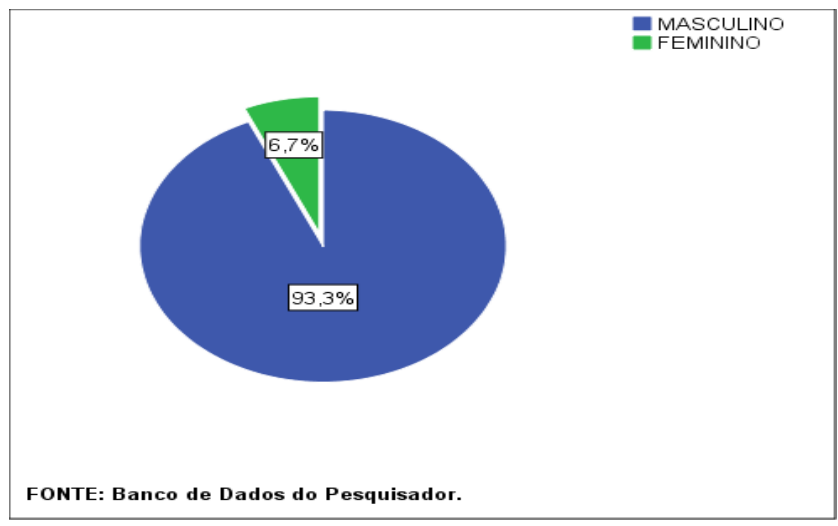

A faixa etária dos indivíduos, dependentes de cocaína/crack, apresentou índice de $43,3 \%$ na categoria entre 30 e 44 anos, seguido de $40 \%$ dos sujeitos com 
idade entre 18 e 29 anos. Os indivíduos com idade entre 45 e 59 anos somam $13,3 \%$ e apenas 3,4\% possuem 60 anos ou mais. (Tabela 1 ).

Tabela 1. Faixa etária dos dependentes de cocaína/crack

\begin{tabular}{l|c|c}
\hline Faixa etária & Frequência & Porcentagem (\%) \\
\hline ENTRE 18 E 29 ANOS & 12 & 40,0 \\
\cline { 2 - 3 } ENTRE 30 E 44 ANOS & 13 & 43,3 \\
\cline { 2 - 3 } ENTRE 45 E 59 ANOS & 4 & 13,3 \\
\cline { 2 - 3 } 60 ANOS OU MAIS & 1 & 3,4 \\
\cline { 2 - 3 } Total & 30 & 100,0 \\
\hline
\end{tabular}

FONTE: Banco de dados do pesquisador.

Referente ao nível de escolaridade dos dependentes, houve maior índice na categoria ensino médio completo, com incidência de $36,7 \%$. A categoria ensino fundamental incompleto ocupa a segunda posição, com índice de $30 \%$ dos entrevistados. O ensino médio incompleto representa $16,7 \%$ dos sujeitos, seguido de $13,3 \%$ com ensino superior completo. Somente 3,3\% dos participantes possuem ensino técnico. Nenhum participante, durante a pesquisa, declarou ser analfabeto (Tabela 2).

Tabela 2. Nível de escolaridade dos dependentes de cocaína/crack

\begin{tabular}{l|c|c}
\hline Nível de escolaridade & Frequência & $\begin{array}{c}\text { Porcentagem } \\
(\%)\end{array}$ \\
\hline Ensino Fundamental Incompleto & 9 & 30,0 \\
\hline Ensino Médio Incompleto & 5 & 16,7 \\
\hline Ensino Médio Completo & 11 & 36,7 \\
\hline Ensino Técnico Completo & 1 & 3,3 \\
\hline Ensino Superior Completo & 4 & 13,3 \\
\hline Total & 30 & 100,0
\end{tabular}

${ }^{*}$ A variável, analfabeto, obteve resultado igual a zero.

Fonte: Banco de Dados do Pesquisador.

Quando questionado sobre a frequência da prática sexual dos participantes, observou-se que a variável de prática sexual, esporádica, obteve o maior índice, com $50 \%$ do total, seguida da prática sexual de, 2 a 3 vezes por semana, com percentual de $33,3 \%$ e prática sexual, 1 vez por semana, com 16,7\%. A variável de, abstenção sexual, obteve resultado nulo. (Tabela 3 ).
Tabela 3. Frequência de prática sexual dos dependentes de cocaína/crack

\begin{tabular}{l|c|c}
\hline \multicolumn{1}{c|}{ Frequência sexual } & Frequência & Porcentagem $(\%)$ \\
\hline 2 A 3 vez por semana & 10 & 33,3 \\
\cline { 2 - 3 } 1 Vez por semana & 5 & 16,7 \\
\cline { 2 - 3 } Esporádica & 15 & 50,0 \\
\cline { 2 - 3 } Total & 30 & 100,0 \\
\hline
\end{tabular}

*A variável abstenção sexual obteve resultado igual a zero. Fonte: Banco de Dados do Pesquisador.

Com relação ao número de parceiros sexuais, 46,7\% dos dependentes de cocaína/crack afirmaram possuir parceiro único, $30 \%$ relataram possuir múltiplos parceiros e $23,3 \%$ dos participantes referiram não possuir parceiro atualmente (Tabela 4).

Tabela 4. Número de parceiros sexuais dos dependentes de cocaína/crack

\begin{tabular}{l|c|c}
\hline Parceiros sexuais & Frequência & Porcentagem (\%) \\
\hline Único parceiro & 14 & 46,7 \\
\hline Múltiplos parceiros & 9 & 30,0 \\
\hline $\begin{array}{l}\text { Não possui parceiro } \\
\text { atualmente }\end{array}$ & 7 & 23,3 \\
\hline Total & 30 & 100,0 \\
\hline
\end{tabular}

Fonte: Banco de Dados do Pesquisador.

Quando questionados sobre a frequência do uso de preservativo, durante as relações sexuais, observou-se que $56,7 \%$ dos indivíduos declararam que não costumam usar, os outros $43,3 \%$ dos participantes referiram fazer uso de proteção, durante as relações sexuais, (Gráfico 2).

Gráfico 2. Frequência de uso de preservativos em relações sexuais entre os dependentes de cocaína/crack

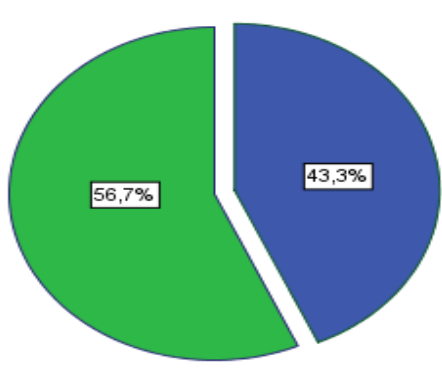

FONTE: Banco de Dados do Pesquisador. 
Com base nas afirmações dos dependentes, participantes da pesquisa, constatou-se que $70 \%$ dos dependentes entrevistados já tiveram prática sexual sob efeito de cocaína e/ou crack e apenas 30\% deles afirmaram que não costumam manter relações sexuais sob efeito das drogas (Gráfico 3).

Gráfico 3. Incidência de relações sexuais dos dependentes sob efeito de cocaína/crack

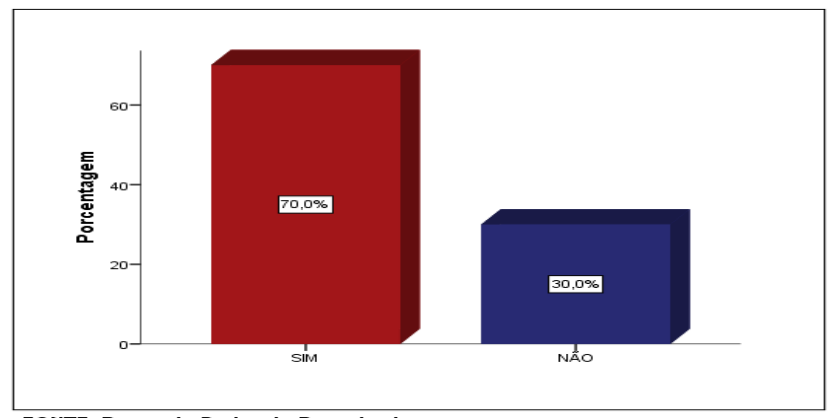

Fonte: Banco de dados do Pesquisador.

No que concerne ao uso de preservativos, $66,7 \%$ dos entrevistados alegaram que já deixaram de fazer uso, por estar sob efeito de cocaína e/ou crack, durante a relação sexual e 33,3\% relataram não deixar de fazer uso de preservativo, ainda que sob efeito das drogas (Gráfico 4).

Gráfico 4. Dependentes de cocaína/crack que deixaram de fazer uso de preservativo por estarem sob o efeito das drogas.

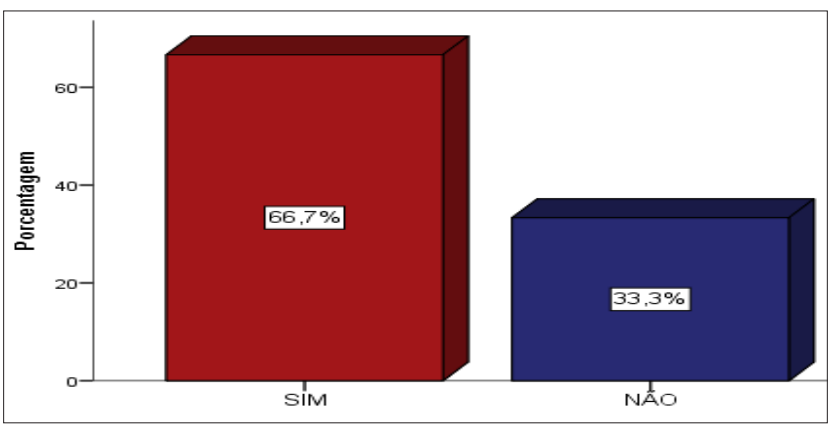

Fonte: Banco de dados do Pesquisador.

Quando questionados quanto à prostituição, como um meio para obtenção de drogas, $76,6 \%$ dos indivíduos referiram nunca ter se prostituído, enquanto que $23,3 \%$ relataram ter usado a prostituição como meio para a obtenção de cocaína e/ou crack (Tabela 5).
Tabela 5. Dependentes de cocaína/crack que já se prostituíram para conseguir as drogas

\begin{tabular}{l|c|c}
\hline \multicolumn{1}{c|}{ Prostituição } & Frequência & Porcentagem (\%) \\
\hline \multirow{2}{*}{ SIM } & 7 & 23,3 \\
\cline { 2 - 3 } NÃO & 23 & 76,7 \\
\cline { 2 - 3 } Total & 30 & 100,0 \\
\hline
\end{tabular}

Fonte: Banco de dados do Pesquisador.

Referente ao histórico de doenças sexualmente transmissíveis, observou-se que $66,6 \%$ dos dependentes de cocaína/crack declararam não possuir histórico de DSTs; $16,7 \%$ relataram ter desenvolvido alguma doença sexualmente transmissível após o início do uso de drogas; $10 \%$ alegaram ter desenvolvido DSTs antes e após o uso de drogas e 6,7\% mencionaram doenças sexualmente transmissíveis antes do uso de drogas (Gráfico 5).

Gráfico 5. Histórico de doenças sexualmente transmissíveis dos dependentes de cocaína/ crack.

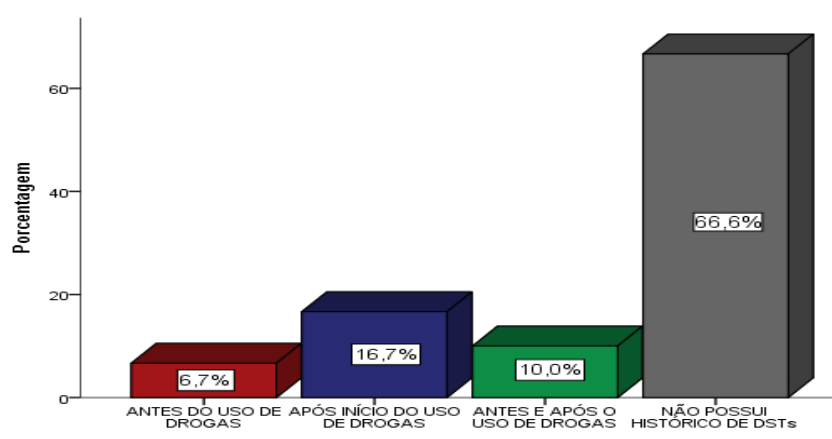

Fonte: Banco de dados do Pesquisador.

Sobre o histórico de doenças, os entrevistados relataram ter desenvolvido um percentual de $10 \%$ para gonorreia; $6,7 \%$ sífilis; 3,3\% para HIV e 13,3\% para outras doenças. A incidência dos que declararam não ter histórico de DSTs foi de 66,7\% (Tabela 6).

Tabela 6. Doença sexualmente transmissível desenvolvida pelos dependentes de cocaína/crack

\begin{tabular}{l|c|c}
\hline \multicolumn{1}{c|}{ DSTs } & Frequência & Porcentagem (\%) \\
\hline Nenhuma & 20 & 66,7 \\
\hline Sífilis & 2 & 6,7 \\
\hline Hiv & 1 & 3,3 \\
\hline Gonorreia & 3 & 10,0 \\
\hline Outras & 4 & 13,3 \\
\hline Total & 30 & 100,0 \\
\hline
\end{tabular}

Fonte: Banco de dados do Pesquisador. 


\section{DISCUSSÃO}

A distribuição dos dados, relacionados aos dependentes de cocaína/crack, evidenciou maior incidência masculina, resultado recorrente na maioria dos estudos sobre o tema. De acordo com Horta ${ }^{14}$, que encontrou resultado semelhante, o fenômeno decorre de fatores históricos e sociais, oriundos das questões de gênero, onde homens possuem uma tendência natural à busca por substâncias psicotrópicas, quando insatisfeitos com aspectos pessoais. As mulheres, em contrapartida, ao buscarem apoio nas relações afetivas, encontram em tal fato uma medida protetiva.

A idade predominante, dos sujeitos entrevistados, foi de 30 a 44 anos (43,3\%), seguida pela faixa etária de 18 a 29 (40\%). Os dados obtidos corroboram com as literaturas vigentes, visto que o estudo realizado por Almeida e colaboradores, ${ }^{15}$ acerca do perfil dos usuários de drogas, identificou que a idade média dos dependentes é de, aproximadamente, 36 anos e que existe declínio do uso, em virtude do avanço cronológico.

Referente ao grau de instrução dos dependentes de cocaína/crack, observou-se que 53,3\% dos participantes da pesquisa possuem, pelo menos, o ensino médio completo, o que contrasta com achados de Garcia, ${ }^{16}$ em que $51 \%$ dos dependentes de crack não possuíam o ensino fundamental completo, sendo que $80 \%$ dos participantes apresentaram níveis baixos de escolaridade.

Tal divergência entre achados sugere que a variável, referente ao nível de escolaridade, pode estar associada ao tipo de população estudada, bem como a região onde a pesquisa foi desenvolvida. Apesar da literatura apontar a maior incidência sobre o baixo índice de escolaridade, entre usuários de drogas, faz-se necessário realizar mais estudos, uma vez que a dependência química está presente em todas as camadas sociais.

Quanto ao comportamento sexual dos entrevistados, $50 \%$ relataram manter prática sexual esporádica, seguido por 33,3\% com prática sexual de 2 a 3 vezes por semana. Quando questionados a respeito do número de parceiros, $46,7 \%$ dos sujeitos declararam possuir parceiro único, enquanto que 30\% alegaram possuir múltiplos parceiros. De acordo com um estudo realizado por Giacomizzi, ${ }^{17}$ 47,7\% dos usuários de drogas declararam possuir parceiro fixo, nos últimos 12 meses, enquanto que $40,7 \%$ referiram ter mais de um parceiro.
A pesquisa desenvolvida por Santos, intitulada Vulnerabilidade e prevalência de HIV em usuários de drogas no Recife ${ }^{18}$ - explicitou que, aproximadamente, $80 \%$ dos entrevistados que declararam possuir parceiro único também mantiveram relações sexuais com parceiros casuais e/ou comerciais, demonstrando assim que o fato do indivíduo alegar manter parceiro único não exclui a possibilidade de um comportamento de risco, expondo também o seu companheiro, uma vez que, segundo estudos de Sousa, ${ }^{19}$ os casais deixam de utilizar preservativo devido ao fator confiança.

No que diz respeito ao uso de preservativos, $56,7 \%$ dos entrevistados mencionaram que não costumam fazer uso e o percentual aumenta para $70 \%$, quando estão sob efeito de drogas. Um estudo realizado por Tuner ${ }^{20}$ afirmou que a maioria das práticas sexuais, desprotegidas, são atribuídas a relacionamentos monogâmicos e ao uso de substâncias psicoativas. Os autores evidenciaram também que o uso de tais substâncias é fator modulador das práticas sexuais, uma vez que interfere na capacidade de ponderação dos riscos, associados às relações desprotegidas.

Quando indagados a respeito da prostituição, como meio de obtenção de drogas, $23,3 \%$ dos sujeitos mencionaram já ter se prostituído. Embora a prostituição seja uma prática comum entre os usuários de cocaína/crack, observou-se que muitos sentem constrangimento em relatar tal fenômeno. Outro ponto a ser considerado é que, a maioria dos entrevistados, na presente pesquisa, pertence ao sexo masculino, contrariando a concepção de que a prostituição, para obtenção de drogas, é exclusiva do sexo feminino. $\mathrm{O}$ estudo realizado por Vernaglia ${ }^{21}$ demonstrou que a utilização do sexo como moeda de troca é algo habitual entre a população usuária de crack e que ocorre em ambos os gêneros.

Referente à prevalência de doenças sexualmente transmissíveis, na população entrevistada, constatou-se que 33,3\% dos indivíduos desenvolveram algum tipo de DST, sendo que $26,7 \%$ deles desenvolveram após o início do uso das drogas, com proporções de desenvolvimento de $10 \%$ para gonorreia; $6,7 \%$ sífilis; $3,3 \%$ HIV e $10 \%$ para outras doenças. É importante salientar que os dependentes, no momento da admissão à Comunidade Terapêutica, foram submetidos a testes para HIV e Sífilis, devendo-se levar em consideração o período da janela imunológica de ambas as patologias. Um estudo realizado por Guimarães ${ }^{22}$ obteve resultado 
análogo, onde $26,2 \%$ dos indivíduos, institucionalizados, relataram o desenvolvimento de alguma doença sexualmente transmissível, sendo que 3,4\% estavam infectados por HIV.

De acordo com o Boletim Epidemiológico de HIV/AIDS 2015, do Ministério da Saúde ${ }^{23}$, no Brasil, cerca de $0,39 \%$ da população geral vive com o HIV e existe uma tendência ao aumento dessa proporção em pessoas que fazem uso de drogas. No ano de 2011, aproximadamente $5,9 \%$ dos usuários de drogas estavam infectados com o vírus e, em 2013, cerca de 5\% dos usuários de crack eram portadores do HIV.

Embora a literatura destaque o aumento da prevalência de sífilis na população geral, conforme Boletim Epidemiológico de Sífilis 201724, sendo 23,8\% para a população brasileira, estudos relacionados à prevalência da doença em usuários de crack revelaram um índice significativamente menor. $\mathrm{O}$ estudo de Nogueira ${ }^{25}$ encontrou o índice de 3,9\% e Ferreira ${ }^{26}$ indicou um percentual de $4,5 \%$, em sua amostra, resultados semelhantes aos do presente estudo.

Tais divergências, entre o Boletim Epidemiológico e os estudos supracitados, sugerem a necessidade do desenvolvimento de outras pesquisas, relacionadas à população usuária de cocaína/crack e a prevalência de sífilis, buscando aqueles que se encontram em situação de uso, uma vez que esses indivíduos não costumam procurar os serviços de saúde, podendo ocorrer a subnotificação.

\section{CONSIDERAÇÕES FINAIS}

A análise do comportamento dos dependentes de cocaína/crack possibilitou a identificação da alta vulnerabilidade dos usuários dessas drogas, em relação à contaminação por doenças sexualmente transmissíveis, uma vez que a adesão ao uso de preservativos é, consideravelmente, baixa e que o efeito das substâncias psicoativas representa um importante fator limitante à utilização de métodos de prevenção às DSTs.

Além do impacto ocasionado pelo uso das substâncias entorpecentes sobre a capacidade de decisão do indivíduo, este fica suscetível à prostituição, como meio para obter as drogas. Tais eventos estão diretamente associados ao ato sexual desprotegido, resultando em uma tendência à contaminação e ao agravo das doenças sexualmente transmissíveis, em meio à população de usuários dessas drogas.
Diante do número reduzido de produções científicas, relacionadas ao uso de cocaína/crack e aos fatores que o permeiam, nota-se que a vulnerabilidade à contaminação por doenças sexualmente transmissíveis é pouco analisada, dificultando, dessa forma, a promoção de medidas preventivas e a otimização dos recursos, a fim de diminuir o índice de contaminação por DSTs.

Os resultados evidenciados nesta pesquisa ratificam a necessidade de abordar os impactos do consumo de cocaína/crack e a vulnerabilidade dessa população à contaminação por DSTs, buscando o fomento de políticas públicas de saúde, capazes de controlar a disseminação dessas DSTs, bem como educação continuada e reinserção desses indivíduos na sociedade.

\section{REFERÊNCIAS BIBLIOGRÁFICAS}

1.BOTELHO, A. P. M.; ROCHA, R. C.; MELO, V. H. Uso e dependência de cocaína/crack na gestação, parto e puerpério. FEMINA. v.41, n.1, 2013.

2.ORGANIZAÇÃO MUNDIALDA SAÚDE. Classificação Estatística Internacional de Doença (CID-10). 7ed. São Paulo: EDUSP, 2012.

3.MARQUES, A. C. P. R.; RIBEIRO, M.; LARANJEIRA, R. R.; ANDRADA, N. C. Abuso e dependência: crack. Rev. Assoc. Med. Bras., v.58, n.2, p.141-153, 2012.

4.PASUCH, C.; OLIVEIRA, M. S. Levantamento sobre o uso de drogas por estudantes do ensino médio: Uma revisão sistemática. Cad. Ter. Ocup. UFSCar. v.22, n. Suplemento Especial, p. 171-83, 2014.

5.UNITED NATIONS OFFICE ON DRUGS AND CRIME, World Drug Report 2016. Vienna: United Nations, 2016.

6.FUNDAÇÃO OSWALDO CRUZ (FIOCRUZ). Pesquisa Nacional sobre o uso de crack: quem são os usuários de crack e/ou similares do Brasil? Quantos são nas capitais brasileiras? Rio de Janeiro: Editora ICICT/FIOCRUZ, 2014.

7.GOOTENBERG, P. Cocaína Andina: El proceso de uma droga global. 1ed. Buenos Aires: Eudeba, 2016. 492p.

8.MUAKAD, I. B. A. COCAÍNA E O CRACK: As Drogas da Morte. Revista da Faculdade de Direito da Universidade de São Paulo, v. 106/107, p. 465-94, jan/dez, 2011/2012.

9.ALVES, V.S.; SAMPAIO, I.M. Atenção à saúde de usuários de álcool e outras drogas no Brasil: Convergência entre a saúde pública e os direitos humanos. RDisan, v.13, n.3, p.9-32, nov/fev, 2013.

10.BRASIL. Secretaria Nacional de Políticas Sobre Drogas. Prevenção ao uso indevido de drogas: Capacitação para 
Conselheiros e Lideranças Comunitárias. 5.ed. Brasília: SENAD, 2013.

11.BRASIL. Secretaria Nacional de Políticas sobre Drogas. Atenção integral na rede de saúde: módulo 5. 7. ed. Brasília: Secretaria Nacional de Políticas sobre Drogas, 2014. 108 p.

12.BRASIL. Legislação e políticas públicas sobre drogas no Brasil. Brasília: Ministério da Justiça, Secretaria Nacional de Políticas sobre Drogas, 2011. 106p.

13.FOSSI, L. B.; GUARESCHI, N. M. F. O modelo de tratamento das comunidades terapêuticas: práticas confessionais na conformação dos sujeitos. Estud. pesqui. psicol., Rio de Janeiro, v.15.n.1, p.94-115, 2015.

14.HORTA, R. L.; HORTA, B.L. ROSSET.; A. P.; HORTA C. L. Perfil dos Usuários de Crack que Buscam Atendimento em Centros de Atenção Psicossocial. Cad. Saúde Pública, Rio de Janeiro, v.27, n.11, p.2263-70, 2011.

15.ALMEIDA, R. A.; ANJOS, U. U.; VIANNA, R. P. T.; PEQUENO, G. A. Perfil dos Usuários de Substâncias Psicoativas de João Pessoa. Rev. Saúde e Debate, Rio de Janeiro, v.38, n.102, p.526-38,2014.

16.GARCIA, E. L.; ZACARIAS, D. G.; WINTER, G.; SONTAG, J. (Re)conhecendo o Perfil do Usuário de Crack de Santa Cruz do Sul. Barbarói, Santa Cruz do Sul, v.36, ed. esp., p.83-95, jan.-jun., 2012.

17.GIACOMIZZI, A. I. Representações sociais das drogas e vulnerabilidade de usuários de CAPs ad em relação às DST/ HIV/AIDS. Estud.pesq.psicolog. Rio de Janeiro, v.11, n.3, dez., 2011.

18.SANTOS, N. T. V. Vulnerabilidade e Prevalência de HIV e Sífilis em Usuários de Drogas no Recife: Resultados de um Estudo Respondent-Driven Sampling. Recife, 2013.145f. Tese (Doutorado em Saúde Pública) Centro de Pesquisas Aggeu Magalhães, Fundação Oswaldo Cruz.

19.SOUSA, L. B.; PINHEIRO, A. K. B. Doenças Transmissíveis na Relação Estável: Perspectivas Para o Cuidado Usando Modelo Sunrise. Rev. Rene. Fortaleza, v.12, n.3, p.478-86, jul.-set., 2011.

20.TURNER, A. K. et al. Physical Victimization and HighRisk Sexual Partners among Illicit Drug-Using Heterosexual Men in New York City. Journal of Urban Health: Bulletin of the New York Academy of Medicine, v.91, n.5, p.95768, 2014.

21.VENAGLIA, T. V. C.; VIEIRA, R. A. M.; CRUZ, M. S. Usuários de Crack em Situação de Rua-Características de Gênero. Ciência \& Saúde Coletiva. Rio de Janeiro, v.20, n.6, p. 1851-59, 2015.

22.GUIMARÃES, R. A.; SILVA, L. N.; FRANÇA, D. D. S.; DEL-RIOS, N. H. A.; CARNEIRO, M. A. S.; TELES,
S. A. Comportamentos de Risco para Doenças Sexualmente Transmissíveis em Usuários de Crack. Rev.Latino-Am. Enfermagem. Goiânia, v.23, n.4, p.628-34, 2015.

23.BRASIL. Ministério da Saúde. Secretaria de Vigilância em Saúde. Expediente Boletim Epidemiológico - Aids e DST. Ano V, n.1 - $27^{\mathrm{a}}$ a $53^{\mathrm{a}}$ - semanas epidemiológicas jul.-dez., 2015.

24.BRASIL, Secretaria de Vigilância em Saúde. Ministério da Saúde. Boletim Epidemiológico SÍFILIS 2017. Brasília. v.48, n. 36, 2017.

25.NOGUEIRA, J. S.; FILHO, C. R. C.; MESQUITA, C. A. M.; SOUZA, E. S. Caracterização dos Usuários Atendidos em um Centro de Testagem e Aconselhamento em Infecções Relacionadas ao Sexo. Revista Saúde e Pesquisa. Maringá, v.10, n. 2, p. 243-250, mai.- ago.,2017.

26.FERREIRA, C. O.; VIANA, A. S. A.; SILVA, A. A.; REZENDE, R. E. A.; GOMES, A. V. T. M.; DAVOGLIO, R.S. Perfil Epidemiológico dos Usuários de um Centro de Testagem e Aconselhamento da Bahia. Revista Baiana de Saúde Pública. v.40, n.2, p. 388-409, abr.-jun., 2016. 